\title{
Tumor-Associated Antigen Expressing Listeria monocytogenes Induces Effective Primary and Memory T-Cell Responses Against Hepatic Colorectal Cancer Metastases
}

\author{
Kelly Olino, MD ${ }^{1}$, Satoshi Wada, MD, $\mathrm{PhD}^{2}$, Barish H. Edil, MD ${ }^{1,2}$, Xiaoyu Pan, $\mathrm{MS}^{2}$, Kristen Meckel, $\mathrm{MS}^{2}$, \\ Walter Weber, $\mathrm{MD}^{1}$, Jill Slansky, $\mathrm{PhD}^{3}$, Koji Tamada, $\mathrm{MD}, \mathrm{PhD}^{4}$, Peter Lauer, $\mathrm{PhD}^{5}$, Dirk Brockstedt, $\mathrm{PhD}^{5}$, \\ Drew Pardoll, MD, PhD $^{2}$, Richard Schulick, $\mathrm{MD}^{1,2}$, and Kiyoshi Yoshimura, MD, $\mathbf{P h D}^{1,2}$ \\ ${ }^{1}$ Department of Surgery, Sidney Kimmel Cancer Center, Johns Hopkins Medical Institutions, Baltimore, MD; \\ ${ }^{2}$ Immunology and Hematopoiesis Division, Department of Medical Oncology, Sidney Kimmel Cancer Center, Johns \\ Hopkins Medical Institutions, Baltimore, MD; ${ }^{3}$ Department of Immunology, University of Colorado Health Sciences \\ Center, Denver, CO; ${ }^{4}$ Department of Otorhinolaryngology, Head and Neck Surgery, University of Maryland School of \\ Medicine, Baltimore, MD; ${ }^{5}$ Aduro BioTech, Berkeley, CA
}

\begin{abstract}
Purpose. Despite advances in therapy for the treatment of metastatic colorectal cancer, many patients die of hepatic disease. Current immunotherapeutic strategies are likely limited by inhibitory signals from the tumor. To successfully eliminate tumor deposits within an organ, an appropriate immunologic milieu to amplify antitumor responses must be developed.

Methods. We used a murine model utilizing the CT26 colon cancer cell line to analyze primary and memory tumor-specific T-cell responses induced by an attenuated actin $\mathrm{A}$ and internalin $\mathrm{B}$ deleted immunodominant tumorassociated antigen expressing strain of Listeria monocytogenes for the treatment of metastatic colorectal cancer.

Results. Treatment of mice bearing established hepatic metastases with this L. monocytogenes strain led to the generation of a strong initial tumor-specific cytotoxic $\mathrm{CD}^{+}$T-cell response that successfully treated $90 \%$ of animals. Tumor antigen-specific central and effector memory $\mathrm{T}$ cells were also generated and protected against
\end{abstract}

Electronic supplementary material The online version of this article (doi:10.1245/s10434-011-2037-0) contains supplementary material, which is available to authorized users.

(C) The Author(s) 2011. This article is published with open access at Springerlink.com

First Received: 6 September 2010;

Published Online: 7 October 2011

K. Yoshimura, $\mathrm{MD}, \mathrm{PhD}$

e-mail: kyoshim6705@gmail.com tumor rechallenge. These cell populations, when measured before and after tumor rechallenge, showed a marked expansion of antigen-specific effector $\mathrm{CD} 8^{+}$effector memory $\mathrm{T}$ cells. This strain of $L$. monocytogenes was able to down-modulate the expression of the immune checkpoint molecule, $\mathrm{PD}-1$, within the tumor microenvironment but had variable effects on CTLA-4 expression.

Conclusions. This L. monocytogenes strain generated a highly effective antitumor T-cell response, providing a basis for the development of this vaccine platform in patients with liver metastases.

Cancer immunotherapy is successful when it eliminates tumor cells and prevents recurrence with the generation of an antigen-specific memory T-cell response. Previous strategies that focused on peptides or cytokine-secreting cancer vaccines, adoptive cytotoxic T-cell transfer, antibody therapy, and dendritic cell therapy have had variable success in generating primary antitumor responses. ${ }^{1-11}$ However, all failed to generate robust acquired immunity and memory $\mathrm{T}$ cells targeted toward tumors, likely as a result of the inability to provide appropriate inflammatory stimuli, so-called danger signals, within the tumor microenvironment. Additionally, effective therapy must overcome multiple mechanisms used by tumors to inhibit antigen-specific immunity including regulatory $\mathrm{T}$ cells (Treg) and inhibitory co-receptor signaling.

Previously, we utilized a genetically attenuated strain of Listeria monocytogenes (with deletion of both actin A and internalin B genes, referred to as LMD), a gram-positive 
bacterium, in a model of hepatic colorectal cancer metastases. We found that LMD generated strong innate antitumor immune responses through activation of NK and NKT cells. A smaller contribution to antitumor effects resulted from cytotoxic $\mathrm{CD} 8^{+} \mathrm{T}$ cells with an interferon- $\gamma$ dominant helper T cell (Th1) phenotype. ${ }^{12-14}$ We developed a new strain of LMD that expressed AH1 (LMD-AH1), the tumor-associated immunodominant antigen of the murine colon cancer cell line CT26 to increase recruitment of the adaptive immune response. ${ }^{15,16}$ Recently, L. monocytogenes was shown to elicit antigen-specific $\mathrm{CD}^{+}{ }^{+}$-cell responses when used as a vaccine vector in breast and ovarian cancer models. ${ }^{17-19}$ By means of this strategy, we elicited strong cytotoxic tumor-specific $\mathrm{CD}^{+}$T-cell responses directly within the liver tumor microenvironment, and achieved lasting cure. We also demonstrated that treatment diminished the expression of PD-1, a key immune checkpoint coinhibitory receptor in $\mathrm{CD} 8^{+} \mathrm{T}$-cell-dendritic cell interactions. This receptor, when expressed on $\mathrm{T}$ cells, renders tumor-infiltrating lymphocytes anergic. ${ }^{20-22} \mathrm{We}$ also observed the generation of a tumor antigen-specific pool of both central and effector memory $\mathrm{T}$ cells in treated mice that survived tumor challenge, with a subsequent expansion of effector memory $\mathrm{CD} 8^{+} \mathrm{T}$ cells in those mice able to reject tumor rechallenge.

\section{METHODS}

\section{Animals, Tumor Cell Line, and Peptides}

BALB/c mice (6-8 weeks old, female) were purchased from the National Cancer Institute and were treated in compliance with the Johns Hopkins Animal Care and Use Committee. CT26 cancer cells were grown in RPMI 1640 medium with $10 \%$ heat-inactivated fetal calf serum (HyClone), $1 \mathrm{mM}$ sodium pyruvate, $2 \mathrm{mM}$ L-glutamine, nonessential amino acids ( $1 \%$ of $100 \times$ stock), penicillin $(100 \mathrm{U} / \mathrm{ml})$, streptomycin $(100 \mathrm{U} / \mathrm{ml})$, and $3 \mu \mathrm{l} \beta$-mercaptoethanol. ${ }^{15}$ AH1 (SPSYVYHQF), a $\mathrm{H}-2 \mathrm{~L}^{\mathrm{d}}$-restricted peptide, and $\beta$-gal (TPHPARIGL) control peptide were synthesized by the Johns Hopkins University Protein/Peptide/DNA facility with $99 \%$ purity. ${ }^{23}$

\section{Listeria Monocytogenes}

LMD was provided by Aduro BioTech. ${ }^{14,24}$ The AH1 peptide expressing strain (LMD-AH1) was constructed from the LMD strain with the AH1 sequence inserted into the gene encoding the actin $\mathrm{A}-$ ovalbumin fusion protein with oligonucleotide-directed polymerase chain reaction. The construct was cloned into the pPL2 integration vector and integrated at the tRNA Arg locus of the bacterial chromosome. ${ }^{25}$ Molecular constructs were confirmed by sequencing.

\section{Murine Hepatic Metastasis Model and Treatment}

Mice were provided isolated hepatic metastases via a hemispleen injection technique (Supplementary Fig. 1). ${ }^{26}$ Treatment doses of LMD or LMD-AH1 were administered intraperitoneally (i.p.) every 3 days at a dose equivalent to one-tenth the experimentally determined lethal dose $(0.1 \times$ $\mathrm{LD}_{50}$ ). A dose of $50 \mathrm{mg} / \mathrm{kg}$ of cyclophosphamide (Sigma) was provided i.p. ${ }^{27}$

\section{Analysis and Isolation of Spleen- and Liver-infiltrating Lymphocytes}

Lymphocytes from livers and spleens were isolated and processed as previously described. ${ }^{14}$ After isolation, cells were blocked with Fc III/II R Antibody (BD PharMingen) and stained with commercially available $\mathrm{CD} 3, \mathrm{CD} 4, \mathrm{CD} 8$, CD62L, CD95, PD-1 (eBioscience). Intracellular staining of CTLA-4 or Foxp3 was done with an intracellular cytokine kit (eBioscience) and analyzed on a FACSCalibur flow cytometer. After lymphocyte isolation, 1:1000 of Golgistop (BD Biosciences) and $20 \mu \mathrm{g} / \mathrm{ml}$ of $\mathrm{AH} 1$ or $\beta$-gal peptide were added $(2: 1000)$ and coincubated at $37^{\circ} \mathrm{C}$ for $5 \mathrm{~h}$, followed by intracellular staining with interferon- $\gamma$ (BD PharMingen). Tetramer staining was performed on liver-infiltrating lymphocytes after magnetic bead $\mathrm{CD}^{+}$ T-cell isolation (Milteny Biotec) and stained with an AH1or $\beta$-gal-specific $\mathrm{L}^{\mathrm{d}}$ tetramer, provided by Jill Slansky.

\section{In Vivo CTL Assay}

In vivo cytotoxic activity of antigen-specific $\mathrm{T}$ cells was assayed as previously described. ${ }^{28}$ The CFSE $^{\text {high }}$-labeled cells were pulsed with $10 \mu \mathrm{g} / \mathrm{ml}$ (1:1000) of AH1 peptide, and CFSE $^{\text {low }}$-labeled cells were pulsed with $10 \mu \mathrm{g} / \mathrm{ml}$ $\beta$-gal control peptide. Forty hours after injection of the CFSE-labeled target cells, the ratio of $\mathrm{CFSE}^{\text {low }}$ to $\mathrm{CFSE}^{\text {high }}$ cells was determined by flow cytometry.

\section{Subcutaneous Flank and Pulmonary Metastases CT26 Tumor Challenge}

For primary challenge and rechallenge experiments, mice were injected with $1 \times 10^{5}$ CT26 cells subcutaneously. Tumor volume was calculated as $0.5\left(\mathrm{~A} \times \mathrm{B}^{2}\right)$, with $\mathrm{B}$ equal to the smaller diameter. Pulmonary metastases were provided via tail vein injection with $5 \times 10^{4}$ for primary challenge or $1 \times 10^{5} \mathrm{CT} 26$ cells for rechallenge experiments. 


\section{AH1 Antigen-Specific CD8 Memory T-Cell Analysis}

Hepatic metastases survivors from each treatment group and tumor-naive mice underwent partial splenectomy. Splenic lymphocytes underwent AH1-specific tetramer and extracellular staining for CD8, CD62L, and CD95 (eBioscience). After 3 days, mice received a flank injection of CT26 tumor cells, and 8 days later underwent completion splenectomy with repeat staining.

\section{Statistical Analysis}

Statistical analyses were performed with GraphPad Prism 5.0. Log-rank analysis was performed for survival experiments. The Mann-Whitney was used for analysis of the AH1 antigen-specific memory $\mathrm{CD} 8^{+} \mathrm{T}$-cell experiment. Two-way analysis of variance (ANOVA) with Bonferroni posttest analysis was performed for all other analyses. A $P$ value of $<0.05$ was considered statistically significant. All experiments were repeated three times, and representative results of each experiment are shown.

\section{RESULTS}

Tumor-Associated Antigen Expressing Listeria Monocytogenes (LMD-AHI) has Enhanced Antitumor Efficacy Against CT26 Metastases

The therapeutic effect of LMD-AH1 was compared to treatment with LMD or no treatment (NT) after hepatic metastases tumor challenge. Mice treated with LMD-AH1 exhibited $90 \%$ survival $(P=0.016$ vs. LMD and $P<0.001$ vs. NT) compared with $40 \%$ survival for mice treated with $\operatorname{LMD}(P=0.002$ vs. NT) (Fig. 1b), consistent with our previously published reported efficacy for LMD. $^{14,26}$

In our initial work, when mice were challenged with CT26 pulmonary metastases and treated with the LMD strain, no mice survived, likely as a result of a lack of a systemically generated effector $\mathrm{CD} 8^{+} \mathrm{T}$-cell response. However, after tail vein injection, $40 \%$ of mice treated with LMD-AH1 survived $(P=0.011)$ (Fig. 1c). This finding was corroborated by Brockstedt et al., who used an AH1A5 peptide expressing strain of LMD to achieve a survival

a
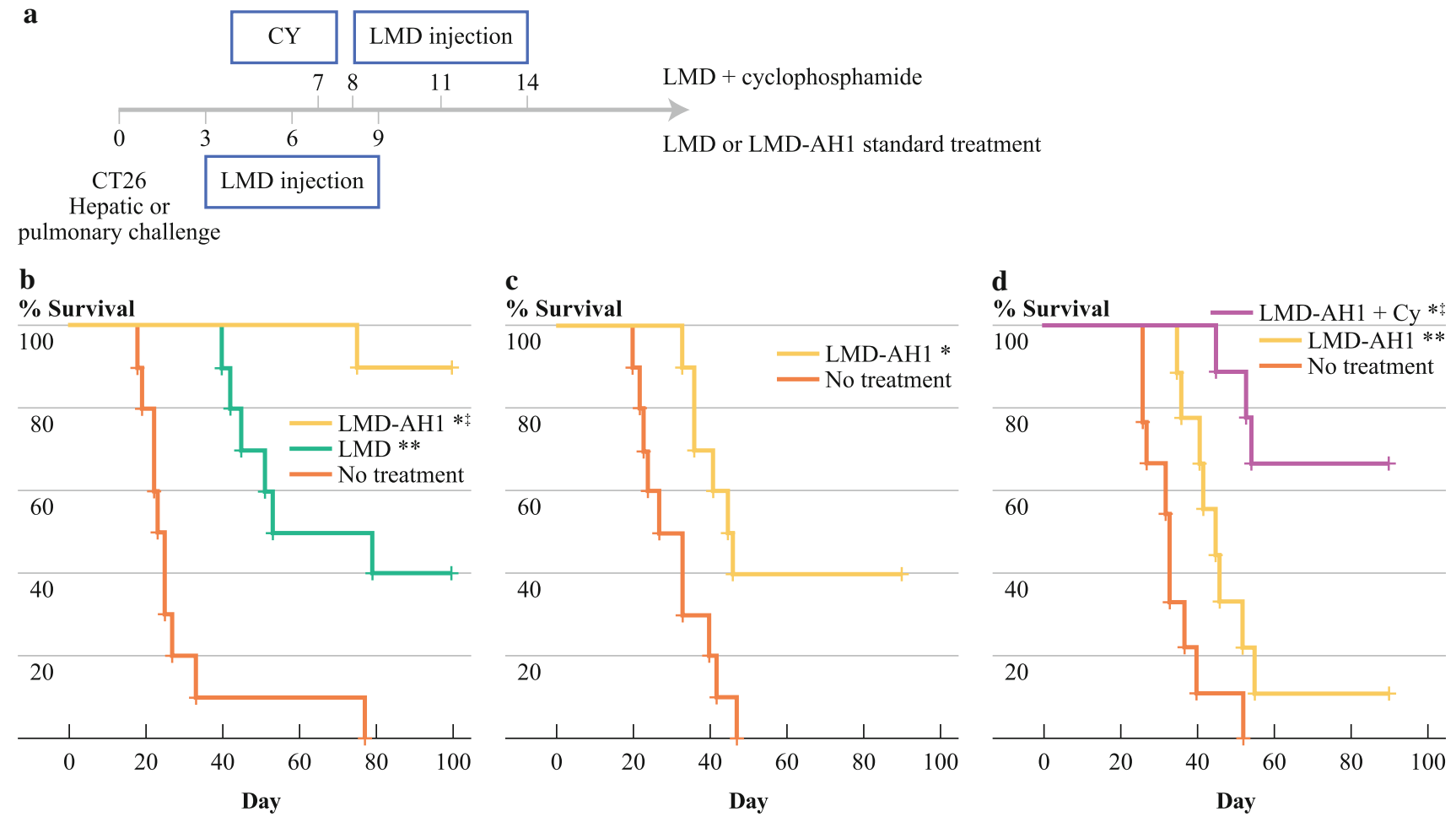

FIG. 1 Treatment with tumor antigen-expressing Listeria (LMDAH1) improves survival. a Treatment protocol for $b-d$. b Balb/c mice were treated with injections of $0.1 \times \mathrm{LD}^{50}$ of LMD-AH1 or LMD on days 3, 6, and 9 after CT26 tumor challenge on day 0 with hemisplenectomy liver metastases model; $n=10$ per group. Logrank test, $* P<0.001$ LMD-AH1 versus NT; $\sharp P=0.016$ LMD-AH1 versus $\mathrm{LMD} ; * * P=0.002 \mathrm{LMD}$ versus NT. c Balb/c mice were

treated with LMD-AH1 or LMD on days 3, 6, and 9 after CT26 tumor challenge via tail vein injection; $n=10$ per group. Log-rank test, $* P=0.011$. d Mice were treated with cyclophosphamide $(\mathrm{Cy})$ on day 7 followed with LMD-AH1 on days 8, 11, and 14 or LMD-AH1 alone; $n=10$ per group. Log-rank test, $\ddagger P=0.006$ LMD$\mathrm{AH} 1+\mathrm{Cy}$ versus LMD-AH1; $* P<0.001 \mathrm{LMD}-\mathrm{AH} 1+\mathrm{Cy}$ versus $\mathrm{NT} ; * * P=0.013$ LMD-AH1 versus NT 
benefit after intravenous CT26 challenge. ${ }^{24}$ Of note, in both the liver and pulmonary metastases model, at the time of completion of the survival experiment, mice that were alive showed no evidence of disease, while those that died earlier all had evidence metastatic disease.

Because Tregs represent a critical inhibitor of tumor immunity, the expression of Foxp3 on $\mathrm{CD}^{+}{ }^{+} \mathrm{T}$ cells was studied within the tumor microenvironment in the liver and on splenocytes. Increased numbers of Foxp3+ Tregs were present in all tumor-challenged groups (Supplementary Fig. 3). Given these findings, we attempted to improve survival of LMD-AH1 treated mice by combining treatment with a Treg inhibitory dose of cyclophosphamide. We previously demonstrated efficacy when combining cyclophosphamide and LMD in a low tumor burden model due to the depletion of Treg cells and a subsequent improvement in the numbers and activation of antigen-specific $\mathrm{CD}^{+}{ }^{+}$T cells. ${ }^{14}$ Given the improved efficacy of the LMDAH1 strain, we waited until a higher tumor burden (macroscopic disease) was present before beginning treatment. Mice provided a single dose of $50 \mathrm{mg} / \mathrm{kg}$ of cyclophosphamide (Cy) on day 7 followed by LMD-AH1 on days 8 , 11 , and 14 had $60 \%$ survival, compared with $10 \%$ for LMD-AH1 alone $(P=0.006$ LMD-AH1 + Cy vs. LMDAH1; $P<0.001$ LMD-AH1 + Cy vs. NT; $P=0.013$ LMD-AH1 vs. NT) (Fig. 1d).

\section{Mice Treated with LMD-AHI Have a Sustained Increase in Antigen-Specific CD $8^{+} \mathrm{T}$ Cells}

To assess tumor antigen-specific $\mathrm{CD}^{+} \mathrm{T}$ cells, we stained isolated liver lymphocytes with an AH1 peptide loaded $\mathrm{L}^{\mathrm{d}}$ tetramer. Those treated with LMD-AH1 had the highest percentage of $\mathrm{CD}^{+}$AH1-specific T cells (1.89\%) compared with LMD $(0.11 \%)$, NT $(0.46 \%)$, or naive $(0.13 \%)$ (Fig. 2a). The peak on day 9 was followed by a lower but steady population through day 11 for LMD-AH1 treated mice (Fig. 2b). In contrast, no discernable peak and a small number of antigen-specific $\mathrm{CD} 8^{+} \mathrm{T}$ cells were found in groups treated with LMD or that received no treatment.

\section{Both Tumor-Bearing and Non-Tumor-Bearing Mice Treated with LMD-AHI Have Higher Interferon- $\gamma$ Expression in $C D 8^{+} T$ Cells}

We hypothesized that treatment with LMD-AH1, even in a tumor-naive mouse, would activate $\mathrm{CD} 8^{+} \mathrm{T}$ cells and direct an antigen-specific adaptive immune response measured by ex vivo stimulation with the AH1 peptide. Tumornaive mice were injected with LMD or LMD-AH1 and $\mathrm{CD}^{+}{ }^{+}$T-cell interferon- $\gamma$ production was measured by intracellular staining. On day 6 (Fig. 2d), mice treated with
LMD-AH1 had more antigen-specific $\mathrm{CD} 8^{+}$T-cell interferon- $\gamma$ expression $(P<0.001$ LMD-AH1 vs. LMD, NT and Naive). Similar results were observed when tumorchallenged mice were treated with LMD-AH1 (Fig. 2d) with peak interferon- $\gamma$ activity observed on day 9 after tumor challenge $(P<0.001$ LMD-AH1 vs. LMD, NT and Naive). These findings also correspond to the peak time of antigen-specific $\mathrm{CD} 8^{+} \mathrm{T}$ cells found on tetramer staining. Mice treated with LMD alone had a later and smaller peak on day 11 , while NT mice lacked interferon- $\gamma$ production, similar to non-tumor-bearing naive mice. Interestingly, when interferon- $\gamma$ production from $\mathrm{CD}^{+}{ }^{+} \mathrm{T}$ cells was examined, there were no differences between LMD-AH1 or LMD treated mice, and only slight increases when compared to NT or naive mice (data not shown). When interleukin-17 expression was examined on $\mathrm{CD} 4^{+} \mathrm{T}$ cells, no differences were observed between any groups (data not shown).

\section{Tumor-Specific CD $8^{+} T$ Cells Isolated from the Livers and Spleens of Mice Treated with LMD-AHI Have the Highest Cytotoxic Killing Ability}

To measure functional differences between lymphocytes from mice treated with LMD-AH1, in vivo CTL assays were performed on days 9 and 11 after hepatic tumor challenge. In Fig. 3a, the mean percentage killing was highest for LMD- AH1 on days 9 and 11 (5.05 and 6.05\%) $(P<0.05$ for LMD-AH1 vs. NT and Naive). In the spleen (Fig. 3b), mean percentage killing was again highest for the LMD-AH1 group on both days peaking on day 11 (1.96 and $5.59 \%)(P<0.05$ for LMD-AH1 vs. LMD; LMD-AH1 vs. naive). Again, because targeted killing was observed for LMD-AH1 treated mice, these results are consistent with the presence of a robust, activated population of immune cells.

Treatment with LMD-AHI Leads to Long-Term Antigen-Specific Memory and Immunity from Tumor Rechallenge

After 12 weeks, mice that survived initial tumor challenge and treatment with LMD-AH1, LMD, or tumor-naive mice underwent partial splenectomy. $\mathrm{CD}^{+} \mathrm{AH} 1$ tetramerspecific effector $\left(\mathrm{CD} 62 \mathrm{~L}^{\text {low }} \mathrm{CD} 95^{\text {high }}\right)$ and central (CD62 $\mathrm{L}^{\text {high }} \mathrm{CD} 95^{\text {high }}$ ) memory $\mathrm{T}$ cells were identified before and after flank challenge with CT26. At both time points, higher percentages of both AH1-specific central and effector memory $\mathrm{T}$ cells were found in mice previously treated with LMD-AH1 (LMD-AH1 vs. naive $P<0.05$ on day 11) (Fig. 4b,c). When mice were stratified by presence or absence of tumor growth, by day 11 , mice that rejected tumor had higher numbers of AH1-specific effector 
$\mathbf{a}$

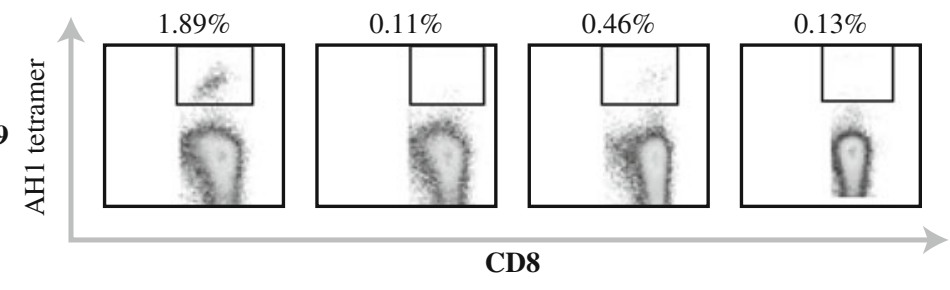

LMD-AH1

LMD

No treatment

Naive

b

Total number CD8+ AH1

specific $\mathrm{T}$ cells

Tumor appearance

c
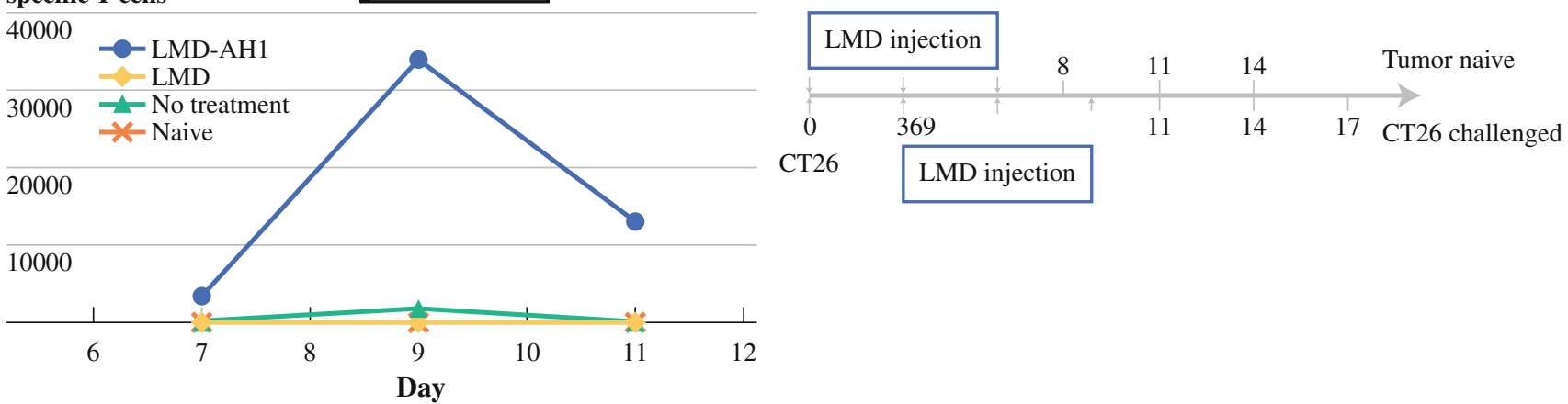

d

Total number

INF-gamma

CD8+ Tcells

15000

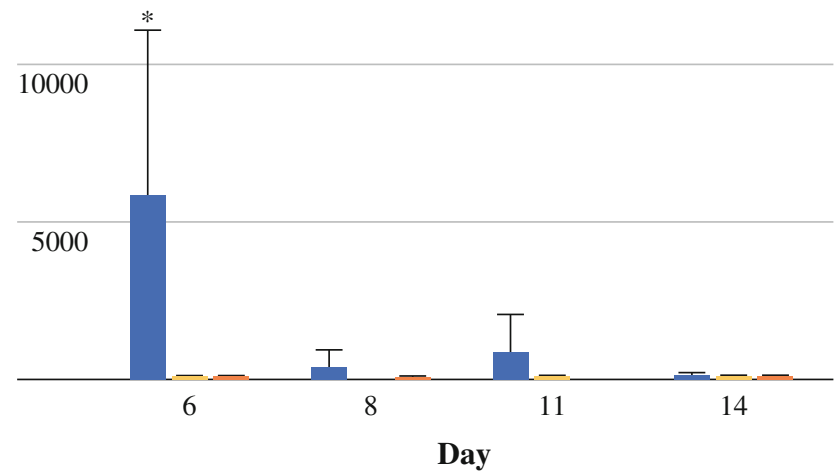

FIG. 2 Mice treated with LMD-AH1 have a high number and sustained population of antigen-specific $\mathrm{CD} 8^{+} \mathrm{T}$ cells with interferon$\gamma$ activity. a Percentage of AH1-specific $\mathrm{CD}^{+} \mathrm{T}$ cells on day 9 in the liver; $n=3$ mice pooled per group. b Total number of AH1-specific $\mathrm{CD}^{+} \mathrm{T}$ cells on days 7, 9, and 11. c Diagram of Listeria injection schedule of tumor-naive mice compared with CT26-challenged mice. d Total number of $\mathrm{CD}^{+} \mathrm{T}$ cells collected from the liver from tumor-

memory $\mathrm{CD}^{+} \mathrm{T}$ cells $(P=0.027)$ and trended toward a greater number of AH1-specific central memory $\mathrm{CD} 8^{+} \mathrm{T}$ cells (Fig. 4b,c). Importantly, this effect was antigen-specific because when total central and effector memory $\mathrm{CD}^{+}{ }^{+} \mathrm{T}$-cell populations were examined there were no differences between treatment groups and no correlation between the absolute number of memory cells and whether the tumor was rejected (Supplementary Fig. 3). e

\begin{tabular}{lll} 
Total number & CT26 challenged & LMD-AH1 \\
INF-gamma & & LMD \\
CD8+ Tcells & & No treatment \\
\hline 15000 & & Naive
\end{tabular}

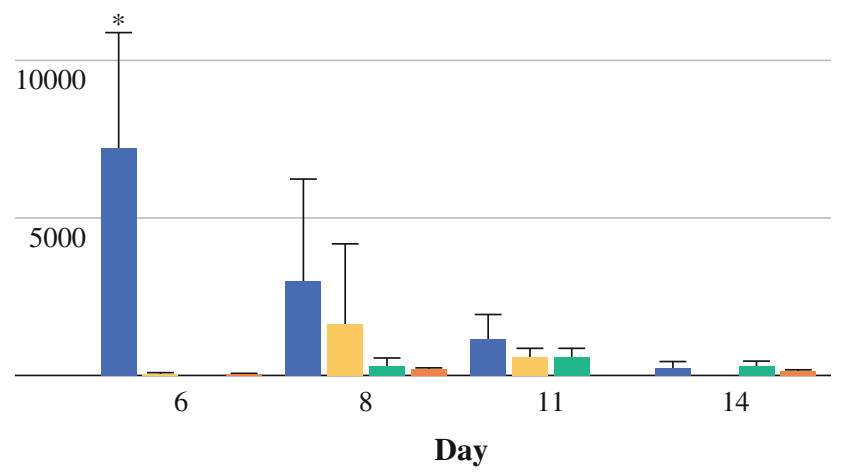

naive or CT26-challenged mice with intracellular staining for interferon- $\gamma$ after AH1 peptide stimulation, $n=3$ mice per group. Two-way ANOVA Bonferroni posttest. $* P<0.001$ LMD-AH1 vs. LMD and naive for day 6 for tumor-naive mice. Two-way ANOVA Bonferroni posttest $* P<0.001$ LMD-AH1 versus LMD, NT and naive for day 9 for CT26-challenged mice. $P=$ NS unless otherwise noted above

To further assess the generation of systemic memory responses after initial rejection of hepatic metastases, animals treated with LMD-AH1 $(n=7)$ or LMD $(n=4)$ that survived 125 days after hepatic tumor challenge were given a rechallenge with $1 \times 10^{5}$ CT26 cells via tail vein injection. Previously treated LMD-AH1 mice had 57\% survival versus $50 \%$ with LMD and $14 \%$ in the tumornaive group $(P=0.044)$ (Fig. 5a). Mice treated with 
a

\% Killing

15

Liver

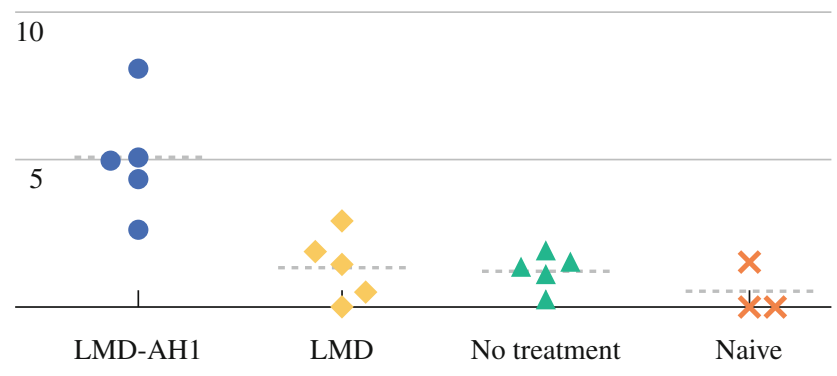

Day 9

\section{b}

$\%$ Killing

15

10

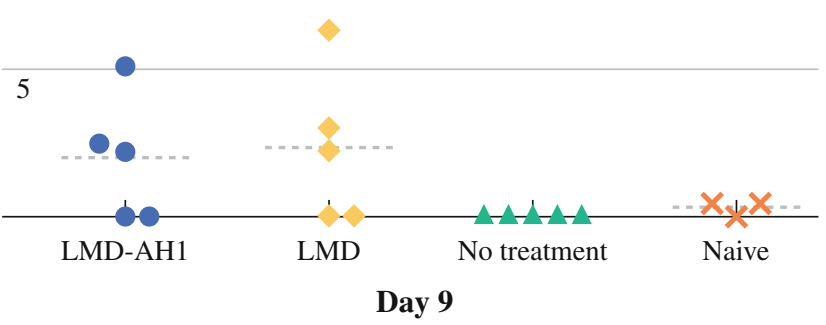

FIG. 3 Mice treated with LMD-AH1 have the highest cytotoxic activity in, in vivo cytotoxic T-cell assay. Mice $(n=5$ per group per day) were challenged with hepatic metastases and treated with LMD or LMD-AH1 on day 3, 6, 9. On day 9 or 11 mice were injected with CFSE ${ }^{\text {high }}$-labeled cells pulsed with AH1 peptide and CFSE ${ }^{\text {low }}$-labeled cells pulsed with $\beta$-gal peptide. a Livers or $\mathbf{b}$ spleens were collected

LMD-AH1 that survived 110 days after initial hepatic metastases challenge were rechallenged with $1 \times 10^{5}$ CT26 tumor cells subcutaneously on the flank (Fig. 5b) and by day 27, 6 of 7 mice previously treated with LMD-AH1 rejected tumor versus 1 of 7 naive mice $(P<0.001)$. When mice given liver metastases that underwent late treatment with LMD-AH1 with cyclophosphamide were rechallenged, 67\% survived $(P<0.01)$ (Fig. 5c).

Treatment with L. monocytogenes Affects PD-1 and CTLA-4 Expression on $\mathrm{CD}^{+} \mathrm{T}$ Cells in Mice with Hepatic Metastases

PD-1 and CTLA-4 are coreceptors expressed on exhausted $\mathrm{CD}^{+} \mathrm{T}$ cells and have been found to be present in large numbers in the tumor microenvironment and are

\begin{tabular}{ll} 
\% Killing & Liver \\
\hline 15 &
\end{tabular}

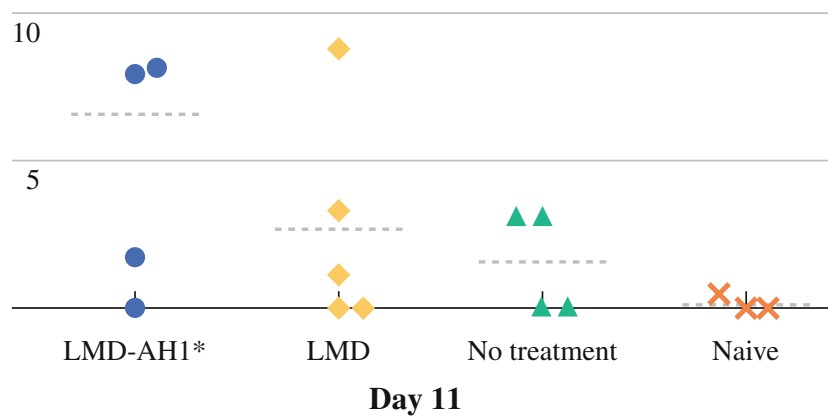

\begin{tabular}{ll}
$\%$ Killing & Spleen \\
\hline 15 &
\end{tabular}

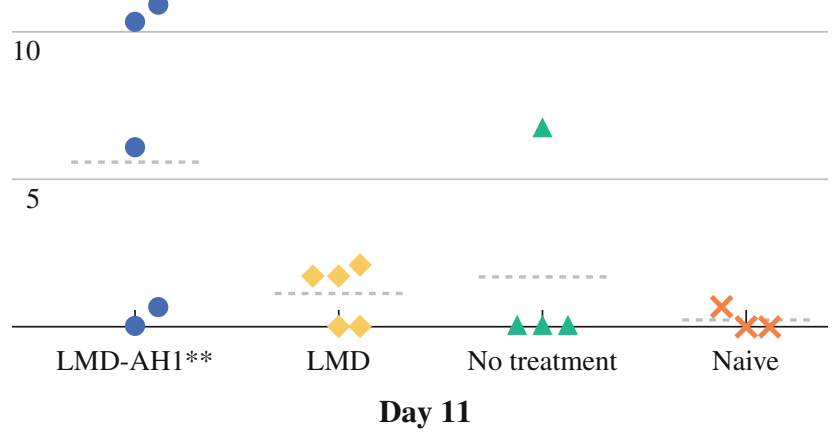

and the ratio of $\mathrm{CFSE}^{\text {high }} / \mathrm{CFSE}^{\text {low }}$ was used to determine percentage killing by flow cytometry. Shown are individual data points with a horizontal line representing the mean. Two-way ANOVA Bonferroni posttest $* P<0.05$ for LMD-AH1 versus NT and naive in the liver and $* * P<0.05$ for LMD-AH1 versus LMD; LMD-AH1 versus naive in the spleen. $P=$ NS unless otherwise noted above

also found in chronically infected animals, rendering $\mathrm{CD} 8^{+}$ $\mathrm{T}$ cells anergic. As LMD is known to cause inflammation we wanted to characterize any change in the expression of these receptors. $\mathrm{CD} 8^{+} \mathrm{T}$ cells in the spleen showed little difference in PD-1 expression between any group even with increasing tumor burden $(P=\mathrm{NS})$ (Fig. 6b). However, in the liver, NT mice showed an increase in both the percentage and absolute number of $\mathrm{CD}^{+} \mathrm{T}$ cell PD-1 expression over time, peaking at day 14 , mirroring the increase in tumor burden (Fig. 6a, b). In groups treated with either LMD or LMD-AH1, little to no increase in PD-1 expression was observed $(P<0.01$ NT vs. LMD-AH1, LMD, and naive in liver on day 14) (Fig. 6b).

CTLA-4 expression was measured on $\mathrm{CD}^{+}{ }^{+} \mathrm{T}$ cells, but when compared to naive mice, all tumor-challenged groups had higher levels of expression even though no group 
FIG. 4 Treatment with LMDAH1 promotes tumor antigenspecific immunologic memory. a Experimental design for parts b-c. Values of the percentage of b $\mathrm{CD}^{+} \mathrm{AH} 1$-specific effector memory (CD62L ${ }^{\text {low }}$ CD95 ${ }^{\text {high }}$ ) or c central memory (CD62 high CD95 $\left.{ }^{\text {high }}\right) \mathrm{T}$ cells are shown by treatment group or as a function of tumor progression or rejection, $n=7$ LMD-AH1, $n=5 \mathrm{LMD}, n=8$ naive. Twoway ANOVA with Bonferroni posttest $* P<0.05$ for LMDAH1 versus naive on day 11 and Mann-Whitney $* P=0.027$ on day 11 for percentage of tumorantigen-specific $\mathrm{CD}^{+}$effector memory T cells. Data for central memory $P=\mathrm{NS}$ $\mathbf{a}$

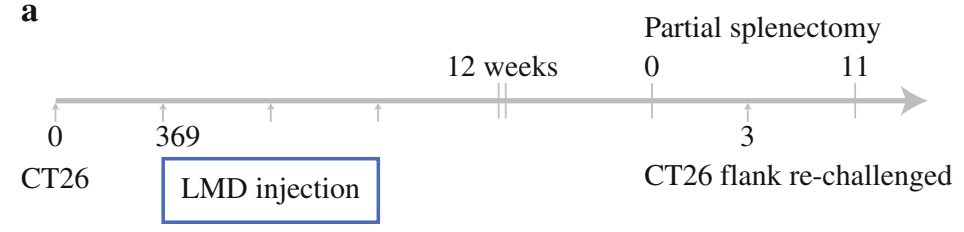

b

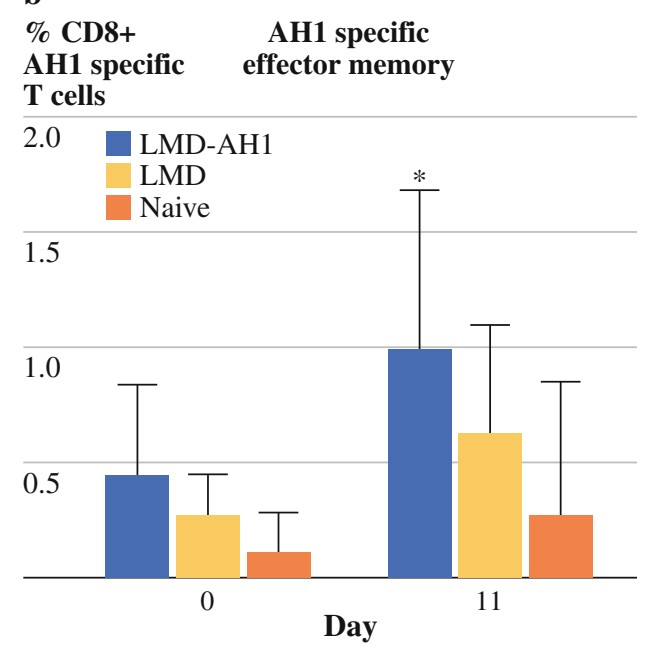

$\begin{array}{lc}\% \text { CD8+ } & \text { AH1 specific } \\ \text { AH1 specific } & \text { effector memory } \\ \text { T cells } & \end{array}$

2.0

Tumor progression

Tumor rejection

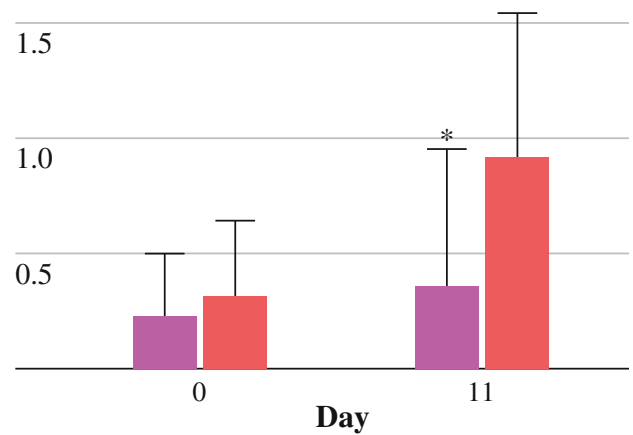

c

\begin{tabular}{l|l}
$\begin{array}{l}\text { \% AH1 } \\
\text { specific }\end{array}$ & \multicolumn{1}{c}{$\begin{array}{c}\text { AH1 specific } \\
\text { central memory }\end{array}$} \\
CD8+ T cells \\
\hline $0.5 \quad$ LMD-AH1 \\
$\begin{array}{ll}\text { LMD } \\
\text { Naive }\end{array}$ \\
\hline
\end{tabular}

$\begin{array}{lc}\% \text { CD8+ } & \text { AH1 specific } \\ \text { AH1 specific } & \text { central memory }\end{array}$

T cells

0.5 Tumor progression

Tumor rejection

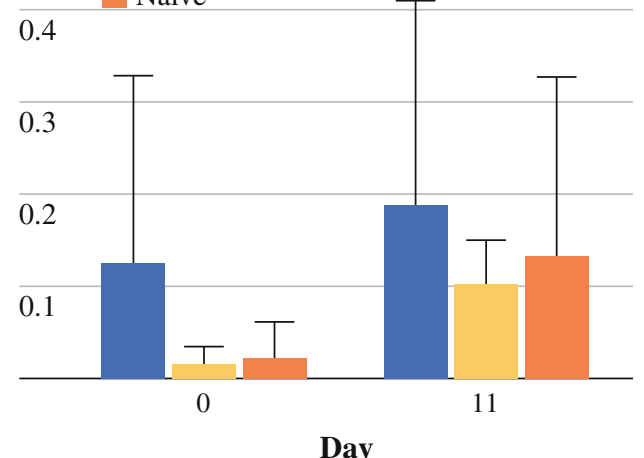

Day

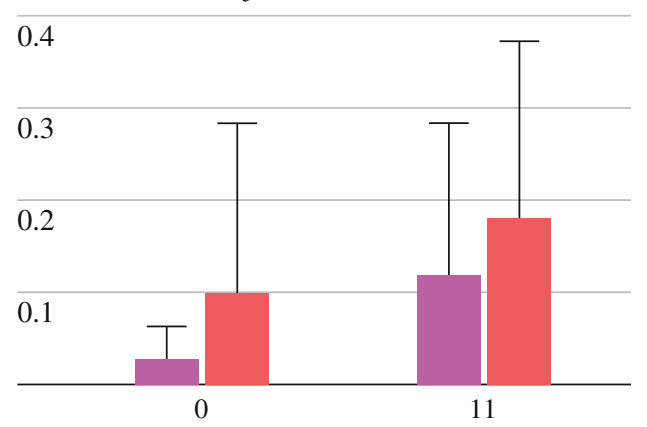

Day reached a statistically significant increase in expression (Fig. 6c). However, consistent with findings by Rowe et al. in a primary attenuated L. monocytogenes infection study, overall CTLA-4 expression on $\mathrm{CD}^{+}{ }^{+} \mathrm{T}$ cells was found to be much higher than on $\mathrm{CD}^{+} \mathrm{T}$ cells in the liver and spleen (Fig. 6d). ${ }^{29}$ In the liver, all groups had higher expression of CTLA-4 than naive mice, with LMD reaching statistically significant expression on day $14(P<0.05)$ (Fig. 6d). On day 14, CTLA-4 expression in the spleens of mice treated with LMD-AH1 had the highest expression among all groups although overall the differences in the spleen were less dramatic $(P<0.05)$. As CTLA-4 expression was increased in the liver tumor microenvironment, blockade of CTLA-4 would be a target to be used in combination with attenuated L. monocytogenes.

\section{DISCUSSION}

Even with advances in liver directed therapies for the treatment of hepatic colorectal metastases, hepatic tumor burden is often rate limiting. We previously described treatment with non-antigen-expressing LMD as an immunotherapeutic treatment for this disease, which could be enhanced either by the depletion of regulatory $\mathrm{T}$ cells with cyclophosphamide or in combination with a GM-CSF secreting cancer vaccine. ${ }^{14,26}$ In our current study, we 


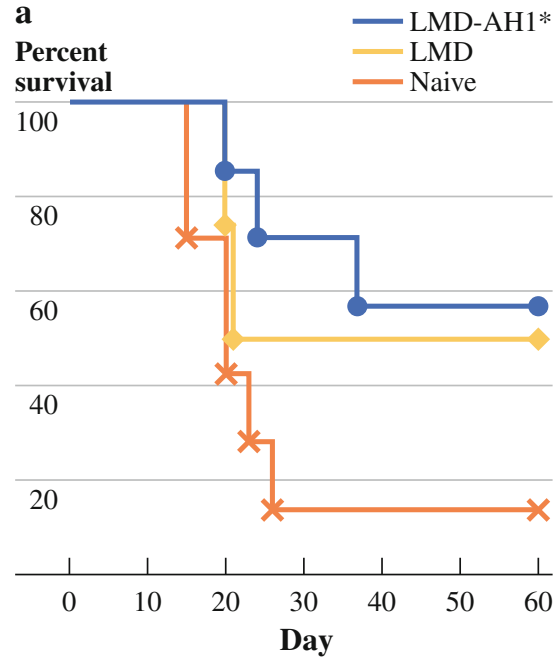

b

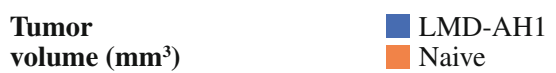

volume $\left(\mathrm{mm}^{3}\right)$

2500

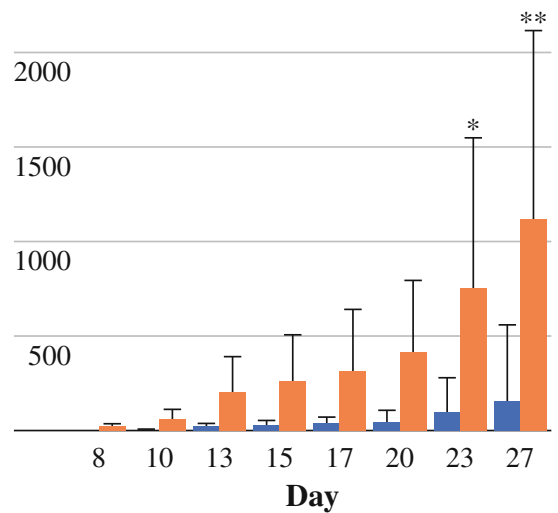

c

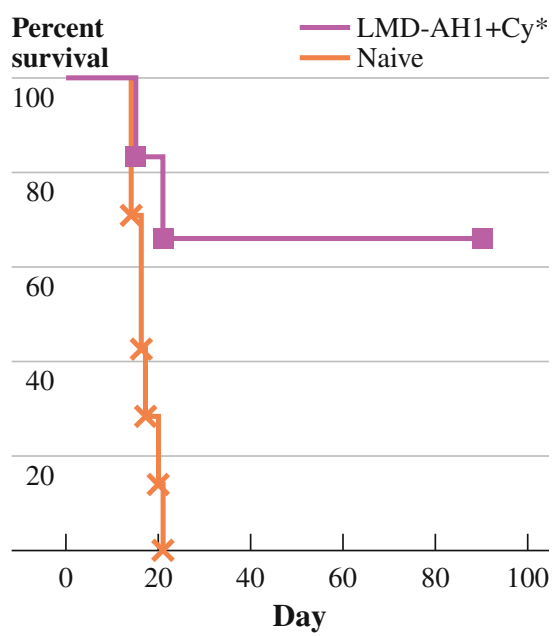

FIG. 5 Treatment with LMD-AH1 protects against tumor rechallenge. a On day 125, previously treated survivors of hepatic metastases were rechallenged with CT26 pulmonary metastases and survival was compared to tumor-naive mice, $n=7$ LMD-AH1, naive group, $n=4$ LMD. Log-rank test $* P=0.044$ for LMD-AH1 versus naive. b On day 110 , survivors of hepatic metastases previously treated with LMD-AH1 were rechallenged with CT26 cells provided through a subcutaneous flank injection, and tumor volume was

demonstrated that use of tumor antigen expressing LMD is more effective in generating an initial effector response by producing $\mathrm{AH} 1$ antigen-specific $\mathrm{CD} 8^{+} \mathrm{T}$ cells in greater numbers than a doubly attenuated strain alone. Furthermore, the activation, as measured by interferon- $\gamma$, and the killing ability of these $\mathrm{CD}^{+} \mathrm{T}$ cells, as shown in the in vivo CTL assay, in both the local tumor environment within the liver and in the systemic circulation were both augmented. In contrast to previous work, this antigen expressing strain was able to enhance survival in both a primary lung and flank tumor challenge study despite the hepatotropic nature of L. monocytogenes. Additionally, LMD-AH1 in combination with cyclophosphamide could cure mice with far greater tumor burden (macroscopic disease) whereas LMD with cyclophosphamide previously cured just microscopic disease. ${ }^{14}$

Both the development of an initial response that eradicates the primary tumor burden and the development of an antigen-specific memory response are important for longterm survival. Although, AH1-specific $\mathrm{CD} 8^{+}$memory $\mathrm{T}$ cells are initially generated by either form of the vaccine, LMD or LMD-AH1. Figures 1-3 show the reliability and overall generation of the initial tumor-specific $\mathrm{CD} 8^{+} \mathrm{T}$ cells was superior in LMD-AH1 treated mice. Mice treated with LMD-AH1 were not only able to survive the original hepatic tumor challenge, but were also able to reject tumor when rechallenged in previously tumor-naive sites.

To specifically investigate the formation of these antigen-specific memory $\mathrm{T}$ cells, we developed a novel compared to tumor-naive mice, $n=7$ for LMD-AH1 group and $n=6$ for NT group. Two-way ANOVA $P=0.034$ with Bonferroni posttest $* P<0.05$ on day 23 and $* * P<0.001$ on day 27 . c On day 140 after hepatic metastases challenge, survivors previously treated with LMD-AH1 + cyclophosphamide were rechallenged with CT26 pulmonary metastases, $n=6$ LMD-AH1 $+\mathrm{Cy}, n=7$ naive. Logrank $* P<0.01 . P=$ NS unless otherwise noted above

technique utilizing repeated surgical intervention. A portion of the spleen was resected before and after tumor rechallenge, giving us a large number of lymphocytes to detect antigen-specific $\mathrm{T}$ cells, which was not technically possible by using circulating blood or other sources. This technique also allowed us to not only to quantify the baseline antigen-specific total effector and central memory populations but also to see whether a change in this response occurred and if this was crucial for tumor rejection. Our data indicate that the presence of higher tumor-specific central and effector memory $\mathrm{CD}^{+}$ T-cell populations was associated with tumor eradication and that antigen-specific effector memory cells underwent expansion.

The development of memory $\mathrm{T}$ cells and the magnitude of cells needed for in vivo responses remain controversial. However, these pathways involve both internal and external stimuli within an inflammatory background with signaling from $\mathrm{CD}^{+} \mathrm{T}$ cells and cytokines released by APCs. ${ }^{30-32}$ When L. monocytogenes was used in another experimental model and compared with adenoviral vectors for tumor antigen delivery, L. monocytogenes showed enhanced memory responses. ${ }^{33}$ Although, this phenomenon may be specific to certain tumor microenvironments, our data supports the liver as a site where the generation of a strong antigen-specific memory response may be dependent on the generation of a Th1 background. ${ }^{33}$ We were able to show that although our treatment causes inflammation, it did not induce a Th17-like background, which 
FIG. 6 Listeria has

differential effects on

coreceptor expression in tumorbearing mice. a Listeria does not cause an upregulation of PD-1 expression on $\mathrm{CD}^{+} \mathrm{T}$ cells. Representative data shown of $\mathrm{CD}^{+} \mathrm{T}$ cells expressing PD1 on day 14 in the liver. b Total $\mathrm{CD}^{+}$T-cell PD-1 expression in the liver and spleen. $* P<0.01$ NT versus LMD-AH1, LMD and naive in liver on day 14 . c Total number of $\mathrm{CD}^{+} \mathrm{T}$ cells expressing the coreceptor CTLA-4 $* P<0.05$ LMD versus naive in liver and spleen. d Total number $\mathrm{CD} 4^{+} \mathrm{T}$ cells expressing CTLA-4 $* P<0.05$ LMD versus LMD-AH1 and $\mathrm{NT} ; * * P<0.05$ naive versus LMD-AH1, LMD, and NT in liver and $* P<0.05$ LMD-AH1 versus LMD, NT, and naive in spleen; $n=3$ mice per group per day. $P=$ NS unless otherwise noted

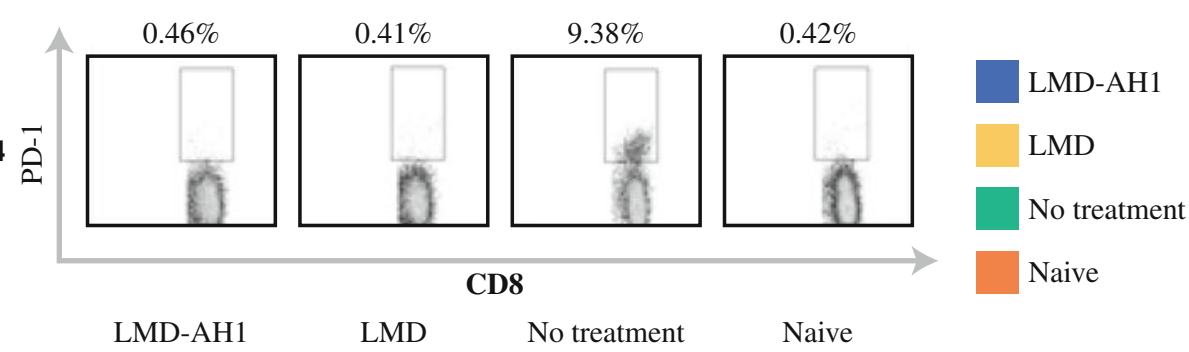

b

Total number

CD8 + PD-1 +

PD-1

Tcells

80000

Liver

\begin{tabular}{l|l} 
& \\
\hline 60000 & \\
\hline 40000 &
\end{tabular}

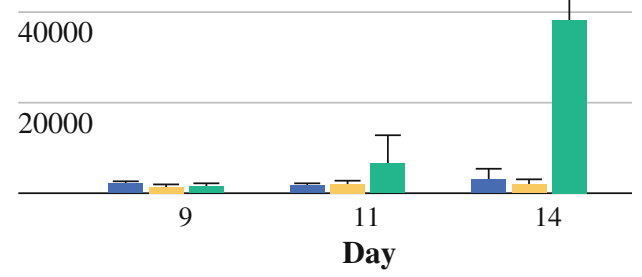

c

\begin{tabular}{lc}
$\begin{array}{l}\text { Total number } \\
\text { CD8 + CTLA-4 } \\
+ \text { Tcells }\end{array}$ & $\begin{array}{c}\text { CTL-4 } \\
\text { Liver }\end{array}$ \\
\hline 15000 & \\
\hline
\end{tabular}

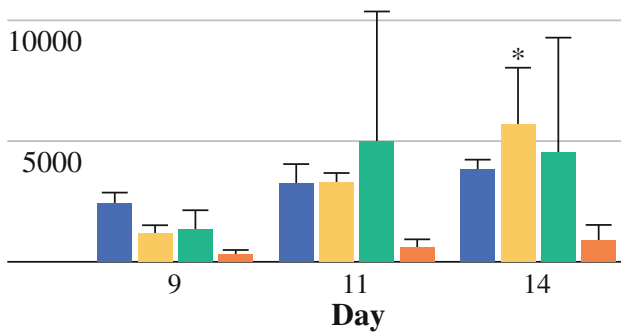

d

\begin{tabular}{lc} 
Total number & CTL-4 \\
CD8 + CTLA-4 & Liver CD4 \\
+ Tcells & \\
\hline
\end{tabular}

80000

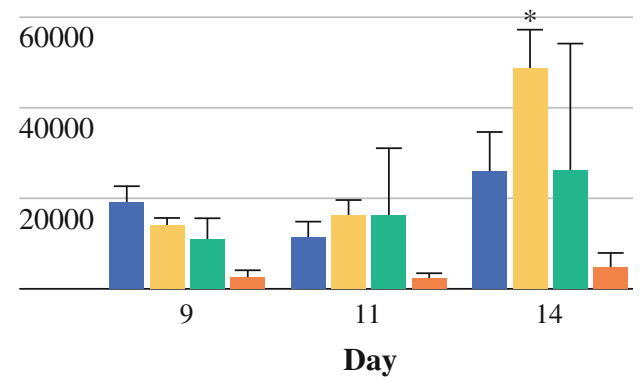

Total number PD-1

CD8 + PD-1 + Spleen

Tcells

80000

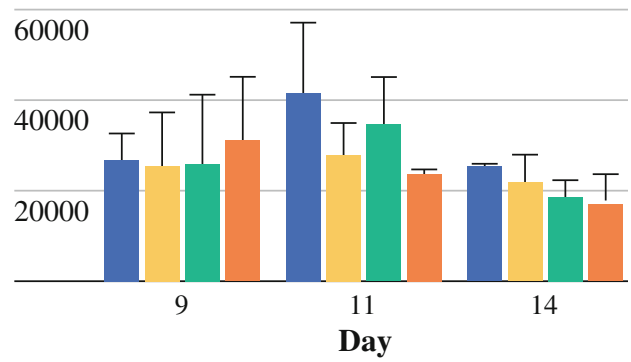

Total number

CTL-4

CD8 + CTLA-4 Spleen

+ Tcells

80000

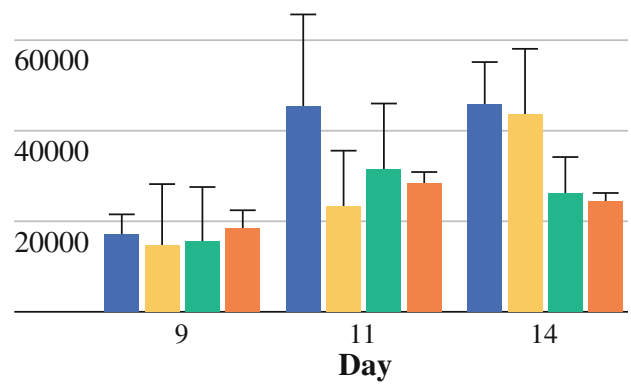

Total number

CD8 + CTLA-4

+ Tcells

400000

CTL-4

Spleen CD4

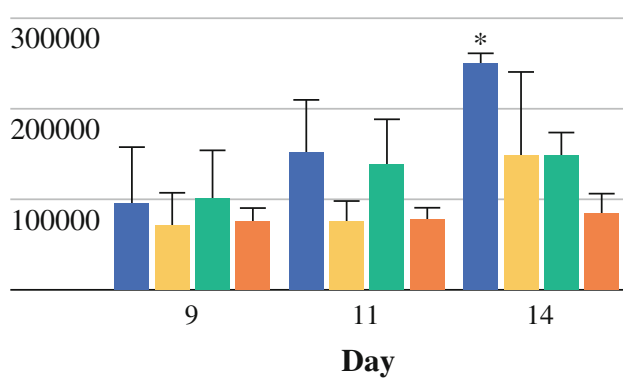


has been implicated in tumor progression. Additionally, the memory responses shown in our work are both durable and specific because they persist without the need to boost or reprime the immune system.

Interestingly, a decrease in the expression of $\mathrm{PD}-1+\mathrm{CD} 8^{+} \mathrm{T}$ cells was observed in mice treated with LMD-AH1. This decrease in PD-1 expression may be either due to the decreased tumor burden causing less induction of PD-1 on tumor infiltrating lymphocytes or possibly may be the direct product of the attenuated $L$. monocytogenes itself through cytokine signaling. L. monocytogenes may also lead to changes in PD-1 expression through modulation of PD-1's receptors, B7-H1 and B7-DC, in the tumor microenvironment. The combination of $L$. monocytogenes with blockade of PD-1 or its receptors may be a viable treatment approach.

Although, we saw less expression of PD-1, a treatment associated decrease in another immune checkpoint receptor, CTLA-4 was not found in our study. To the contrary, we found an increase in CTLA- 4 expression on both $\mathrm{CD}^{+}$ and $\mathrm{CD} 8^{+} \mathrm{T}$-cell populations in all groups challenged with tumor regardless of treatment amongst liver lymphocytes, while little difference was found in the spleen. Given the differences between CTLA- 4 and PD- 1 in their location and temporal expression, these pathways may be differentially affected by our treatment. ${ }^{34,35}$ The increased CTLA-4 expression is important because just as the presence of tumor led to an increase in Foxp3+Tregs, providing a target for combinatorial treatment with cyclophosphamide, one could hypothesize that CTLA-4 would be an additional candidate for blockade when using our vaccine. This hypothesis is supported by previously published findings. ${ }^{30,36}$ Rowe et al. showed that blockade of CTLA-4 led to augmented antigen-specific $\mathrm{CD}^{+}$and $\mathrm{CD} 8^{+} \mathrm{T}$-cell responses directed toward an attenuated strain of L. monocytogenes during primary infection. ${ }^{29}$ Pedicord et al. reported that a single dose of anti-CTLA-4 antibody, when administered with Listeria monocytogenes, enhanced bacterial clearance upon rechallenge as a result of an increase in the production of effector memory $\mathrm{T}$ cells. ${ }^{37}$ Conceivably, the blockade of CTLA-4 could increase antigen-specific responses to tumor-associated antigens delivered by L. monocytogenes allowing for stronger initial antitumor responses or for smaller doses of antigen expressing L. monocytogenes to be administered, thus increasing the safety profile with the use of this bacterium. The initial enhanced response combined with increased effector memory $\mathrm{T}$ cells, could not only lead to improved initial tumor eradication but also to the prevention of recurrent disease.

Although, the identification of cancer associated antigens for targeted therapies has progressed, any successful treatment will need to overcome the barriers presented by
MHC I downregulation, and coreceptor signaling between tumor cells, T cells and APCs leading to continued immune evasion. In our study, the efficacy shown using this genetically attenuated strain of AH1 expressing L. monocytogenes suggests that it can be engineered to synthesize a sufficient amount of tumor-associated antigen through a strong promoter. Additionally, other groups have shown that $L$. monocytogenes can be engineered to express polyvalent tumor-specific antigens. ${ }^{19,36}$ Despite this, advances in genetic engineering of $L$. monocytogenes are needed to determine the relative importance of virulence factors to enable toll-like receptor signaling while maintaining the ability to reliably deliver antigen.

In summary, we have shown that tumor-associated antigen expressing genetically attenuated Listeria monocytogenes used in a murine model of colorectal cancer metastasis is effective to initially treat and subsequently prevent tumor recurrence. This efficacy was demonstrated by inducing three major immune mechanisms: innate, adaptive, and tumor-specific immune memory. The success in this preclinical model continues to show promise in the development of L. monocytogenes-based treatment platforms into effective vaccines in human clinical trials and in finding novel combinatorial regimens to further enhance its observed efficacy.

ACKNOWLEDGMENT Supported in part by NIH grants T32 DK007713-14 (to K.O.), K23 CA104160 (to R.S. and K.Y.), and R01 CA112160 (to R.S. and K.Y.), and Swiss National Science Foundation grant PBBSB-118840 (to W.W.). P.L. and D.B. are employees of Aduro BioTech, which develops Listeria monocytogenes-based vaccines. D.P., R.S., K.Y., and D.B. hold a patent on Listeria-induced immunorecruitment and activation, and methods of use thereof.

OPEN ACCESS This article is distributed under the terms of the Creative Commons Attribution Noncommercial License which permits any noncommercial use, distribution, and reproduction in any medium, provided the original author(s) and source are credited.

\section{REFERENCES}

1. Hueman MT, Dehqanzada ZA, Novak TE, et al. Phase I clinical trial of a HER-2/neu peptide (E75) vaccine for the prevention of prostate-specific antigen recurrence in high-risk prostate cancer patients. Clin Cancer Res. 2005;11:7470-9.

2. Jaffee EM, Hruban RH, Biedrzycki B, et al. Novel allogeneic granulocyte-macrophage colony-stimulating factor-secreting tumor vaccine for pancreatic cancer: a phase I trial of safety and immune activation. J Clin Oncol. 2001;19:145-56.

3. Johnson LE, Frye TP, Arnot AR, et al. Safety and immunological efficacy of a prostate cancer plasmid DNA vaccine encoding prostatic acid phosphatase (PAP). Vaccine. 2006;24:293-303.

4. Peoples GE, Holmes JP, Hueman MT, et al. Combined clinical trial results of a HER2/neu (E75) vaccine for the prevention of recurrence in high-risk breast cancer patients: U.S. Military Cancer Institute Clinical Trials Group Study I-01 and I-02. Clin Cancer Res. 2008;14:797-803. 
5. Ramanathan RK, Lee KM, McKolanis J, et al. Phase I study of a MUC1 vaccine composed of different doses of MUC1 peptide with SB-AS2 adjuvant in resected and locally advanced pancreatic cancer. Cancer Immunol Immunother. 2005;54:254-64.

6. Araki A, Hazama S, Yoshimura K, Yoshino S, Iizuka N, Oka M. Tumor secreting high levels of IL-15 induces specific immunity to low immunogenic colon adenocarcinoma via $\mathrm{CD} 8^{+} \mathrm{T}$ cells. Int J Mol Med. 2004;14:571-6.

7. Kawaoka T, Oka M, Takashima M, et al. Adoptive immunotherapy for pancreatic cancer: cytotoxic $\mathrm{T}$ lymphocytes stimulated by the MUC1-expressing human pancreatic cancer cell line YPK-1. Oncology Rep. 2008;20:155-63.

8. Kondo H, Hazama S, Kawaoka T, et al. Adoptive immunotherapy for pancreatic cancer using MUC1 peptide-pulsed dendritic cells and activated T lymphocytes. Anticancer Res. 2008;28:379-87.

9. Yoshimura K, Hazama S, lizuka N, et al. Successful immunogene therapy using colon cancer cells (colon 26) transfected with plasmid vector containing mature interleukin-18 cDNA and the Igkappa leader sequence. Cancer Gene Ther. 2001;8:9-16.

10. Dudley ME, Yang JC, Sherry R, et al. Adoptive cell therapy for patients with metastatic melanoma: evaluation of intensive myeloablative chemoradiation preparative regimens. J Clin Oncol. 2008;26:5233-9.

11. Hodi FS, Mihm MC, Soiffer RJ, et al. Biologic activity of cytotoxic T lymphocyte-associated antigen 4 antibody blockade in previously vaccinated metastatic melanoma and ovarian carcinoma patients. Proc Natl Acad Sci U S A. 2003;100:4712-7.

12. Chan $\mathrm{CW}$, Crafton $\mathrm{E}, \mathrm{Fan} \mathrm{HN}$, et al. Interferon-producing killer dendritic cells provide a link between innate and adaptive immunity. Nat Med. 2006;12:207-13.

13. Pletneva M, Fan H, Park JJ, et al. IFN-producing killer dendritic cells are antigen-presenting cells endowed with T-cell crosspriming capacity. Cancer Res. 2009;69:6607-14.

14. Yoshimura K, Laird LS, Chia CY, et al. Live attenuated Listeria monocytogenes effectively treats hepatic colorectal cancer metastases and is strongly enhanced by depletion of regulatory $\mathrm{T}$ cells. Cancer Res. 2007;67:10058-66.

15. Griswold DP, Corbett TH. A colon tumor model for anticancer agent evaluation. Cancer. 1975;36:2441-4.

16. Corbett TH, Griswold DP Jr, Roberts BJ, Peckham JC, Schabel FM Jr. Tumor induction relationships in development of transplantable cancers of the colon in mice for chemotherapy assays, with a note on carcinogen structure. Cancer Res. 1975;35:24349.

17. Kim SH, Castro F, Paterson Y, Gravekamp C. High efficacy of a Listeria-based vaccine against metastatic breast cancer reveals a dual mode of action. Cancer Res. 2009;69:5860-6.

18. Seavey MM, Pan ZK, Maciag PC, et al. A novel human Her-2/ neu chimeric molecule expressed by Listeria monocytogenes can elicit potent HLA-A2 restricted CD8-positive T cell responses and impact the growth and spread of Her-2/neu-positive breast tumors. Clin Cancer Res. 2009;15:924-32.

19. Sinnathamby G, Lauer P, Zerfass J, et al. priming and activation of human ovarian and breast cancer-specific $\mathrm{CD} 8^{+} \mathrm{T}$ cells by polyvalent Listeria monocytogenes-based vaccines. J Immunother. 2009;32:856-69.

20. Francisco LM, Salinas VH, Brown KE, et al. PD-L1 regulates the development, maintenance, and function of induced regulatory $\mathrm{T}$ cells. J Exp Med. (in press).

21. Freeman GJ, Long AJ, Iwai Y, et al. Engagement of the PD-1 immunoinhibitory receptor by a novel B7 family member leads to negative regulation of lymphocyte activation. $J$ Exp Med. 2000;192:1027-34.

22. Sharpe AH, Wherry EJ, Ahmed R, Freeman GJ. The function of programmed cell death 1 and its ligands in regulating autoimmunity and infection. Nat Immunol. 2007;8:239-45.

23. Huang AY, Gulden PH, Woods AS, et al. The immunodominant major histocompatibility complex class I-restricted antigen of a murine colon tumor derives from an endogenous retroviral gene product. Proc Natl Acad Sci U S A. 1996;93:9730-5.

24. Brockstedt DG, Giedlin MA, Leong ML, et al. Listeria-based cancer vaccines that segregate immunogenicity from toxicity. Proc Natl Acad Sci U S A. 2004;101:13832-7.

25. Lauer P, Chow MY, Loessner MJ, Portnoy DA, Calendar R. Construction, characterization, and use of two Listeria monocytogenes site-specific phage integration vectors. $J$ Bacteriol. 2002;184:4177-86.

26. Yoshimura K, Jain A, Allen HE, et al. Selective targeting of antitumor immune responses with engineered live-attenuated Listeria monocytogenes. Cancer Res. 2006;66:1096-104.

27. Wada S, Yoshimura K, Hipkiss EL, et al. Cyclophosphamide augments antitumor immunity: studies in an autochthonous prostate cancer model. Cancer Res. 2009;69:4309-18.

28. Mueller SN, Jones CM, Smith CM, Heath WR, Carbone FR. Rapid cytotoxic T lymphocyte activation occurs in the draining lymph nodes after cutaneous herpes simplex virus infection as a result of early antigen presentation and not the presence of virus. J Exp Med. 2002;195:651-6.

29. Rowe JH, Johanns TM, Ertelt JM, Lai JC, Way SS. Cytotoxic T-lymphocyte antigen 4 blockade augments the T-cell response primed by attenuated Listeria monocytogenes resulting in more rapid clearance of virulent bacterial challenge. Immunology. 2009;128:e471-8.

30. Janssen EM, Lemmens EE, Wolfe T, Christen U, von Herrath $\mathrm{MG}$, Schoenberger SP. CD4 ${ }^{+} \mathrm{T}$ cells are required for secondary expansion and memory in $\mathrm{CD} 8^{+} \mathrm{T}$ lymphocytes. Nature. 2003; 421:852-6.

31. Obar JJ, Molloy MJ, Jellison ER, et al. $\mathrm{CD}^{+} \mathrm{T}$ cell regulation of CD25 expression controls development of short-lived effector $\mathrm{CD}^{+} \mathrm{T}$ cells in primary and secondary responses. Proc Natl Acad Sci U S A. 2010;107:193-8.

32. Stemberger C, Huster KM, Koffler M, et al. A single naive CD8 ${ }^{+}$ $\mathrm{T}$ cell precursor can develop into diverse effector and memory subsets. Immunity. 2007;27:985-97.

33. Stark FC, Sad S, Krishnan L. Intracellular bacterial vectors that induce $\mathrm{CD} 8(+) \mathrm{T}$ cells with similar cytolytic abilities but disparate memory phenotypes provide contrasting tumor protection. Cancer Res. 2009;69:4327-34.

34. Fife BT, Bluestone JA. Control of peripheral T-cell tolerance and autoimmunity via the CTLA-4 and PD-1 pathways. Immunol Rev. 2008;224:166-82.

35. Fife BT, Pauken KE, Eagar TN, et al. Interactions between PD-1 and PD-L1 promote tolerance by blocking the TCR-induced stop signal. Nat Immunol. 2009;10:1185-92.

36. Brockstedt DG, Dubensky TW. Promises and challenges for the development of Listeria monocytogenes-based immunotherapies. Exp Rev Vaccines. 2008;7:1069-84.

37. Pedicord VA, Montalvo W, Leiner IM, Allison JP. Single dose of anti-CTLA-4 enhances $\mathrm{CD}^{+}{ }^{+} \mathrm{T}$-cell memory formation, function, and maintenance. Proc Natl Acad Sci U S A. 2011;108:266-71. 